\title{
The diverse roles of FRO family metalloreductases in iron and copper homeostasis
}

\section{Anshika Jain, Grandon T. Wilson and Erin L. Connolly*}

Department of Biological Sciences, University of South Carolina, Columbia, SC, USA

\section{Edited by:}

Marta Wilton Vasconcelos, Universidade Católica Portuguesa, Portugal

\section{Reviewed by:}

Graziano Zocchi, Università degli Studi di Milano, Italy

Khurram Bashir, RIKEN, Japan

\section{${ }^{*}$ Correspondence:}

Erin L. Connolly, Department of Biological Sciences, University of South Carolina, 715 Sumter Street, Columbia, SC 29208, USA e-mail: erinc@biol.sc.edu

Iron and copper are essential for plants and are important for the function of a number of protein complexes involved in photosynthesis and respiration. As the molecular mechanisms that control uptake, trafficking and storage of these nutrients emerge, the importance of metalloreductase-catalyzed reactions in iron and copper metabolism has become clear. This review focuses on the ferric reductase oxidase (FRO) family of metalloreductases in plants and highlights new insights into the roles of FRO family members in metal homeostasis. Arabidopsis FRO2 was first identified as the ferric chelate reductase that reduces ferric iron-chelates at the root surface-rhizosphere interface. The resulting ferrous iron is subsequently transported across the plasma membrane of root epidermal cells by the ferrous iron transporter, IRT1. Recent work has shown that two other members of the FRO family (FRO4 and FRO5) function redundantly to reduce copper to facilitate its uptake from the soil. In addition, FROs appear to play important roles in subcellular compartmentalization of iron as $\mathrm{FRO7}$ is known to contribute to delivery of iron to chloroplasts while mitochondrial family members FRO3 and FRO8 are hypothesized to influence mitochondrial metal ion homeostasis. Finally, recent studies have underscored the importance of plasma membrane-localized ferric reductase activity in leaves for photosynthetic efficiency. Taken together, these studies highlight a number of diverse roles for FROs in both iron and copper metabolism in plants.

Keywords: ferric reductase oxidase, metalloreductase, iron, copper, plant

\section{INTRODUCTION}

Iron $(\mathrm{Fe})$ is essential for plants and is required for the function of a large number of enzymes involved in photosynthesis, respiration and a number of other processes. Iron's utility in myriad biochemical processes stems from its ability to readily accept and donate electrons. It is most often associated with protein complexes either as a component of heme or Fe-S clusters. The ability of Fe to participate in electron transfer reactions is nevertheless problematic as well, since $\mathrm{Fe}^{3+}$ and $\mathrm{Fe}^{2+}$ are able to participate in the generation of the highly reactive hydroxyl radical (Halliwell and Gutteridge, 1992). As a result, it is critical that cells carefully control cellular Fe metabolism.

Iron limits plant growth in many soil types despite the fact that it is usually quite abundant. This is due to the fact that ferric iron is very poorly soluble in aerobic soils at neutral to basic $\mathrm{pH}$. In the presence of oxygen, iron precipitates into insoluble $\mathrm{Fe}(\mathrm{III})$-oxyhydroxide complexes. Thus, the molecular mechanisms utilized by plants for iron acquisition often include a first step that solubilizes ferric iron followed by a second step in which iron is transported from the soil and into root cells. Plants have evolved two types of strategies to combat iron deficiency. Strategy I is a reduction-based method used by all dicots and non-grass monocots while strategy II is used by grass species and involves chelation of ferric iron followed by uptake (Guerinot and Yi, 1994).

In response to iron deficiency, strategy I plants engage in a three stage process to acquire iron. First, the surrounding rhizosphere is acidified via proton extrusion by a root plasma membrane-localized proton ATPase, AHA2 (Arabidopsis $\mathrm{H}^{+}$ATPase 2; Santi and Schmidt, 2009). This serves to increase solubility of ferric iron complexes. Ferric iron chelates are then reduced to ferrous iron by FRO2 (ferric reductase oxidase 2) and $\mathrm{Fe}^{2+}$ ions are subsequently taken up into root cells by the divalent metal transporter, IRT1 (iron regulated transporter 1; Eide et al., 1996; Yi and Guerinot, 1996; Robinson et al., 1999; Vert et al., 2002). In contrast, strategy II plants secrete phytosiderophores (PSs), such as mugineic acid, which bind to ferric iron with high affinity (Walker and Connolly, 2008). The resulting Fe(III)-PS complexes are transported across the root plasma membrane via the yellow stripe1 (YS1) iron transporter (Curie et al., 2001).

In this review, we focus on the roles of the FRO family of metalloreductases in reduction of iron and copper in plants. To this end, we briefly review what is known about reduction of iron at the root surface and highlight new work that has demonstrated a role for FRO family members in reduction of copper for uptake by plants. In addition, we focus on the emerging roles of FROs in trafficking of iron to subcellular compartments.

\section{THE FRO FAMILY OF METALLOREDUCTASES}

The reduction of ferric iron to ferrous iron at the root surface is a process that has been well documented and characterized across several plant species including Arabidopsis (Yi and Guerinot, 1996), pea (Waters et al., 2002), and tomato (Li et al., 2004), as well as the green alga Chlamydomonas reinhardtii (Eckhardt and Buckhout, 
1998). The first plant metalloreductase gene was cloned from Arabidopsis (Robinson et al., 1999). FRO2 was identified based on its sequence similarity to the yeast ferric reductase, FRE1, as well as to a subunit of the human NADPH oxidase, gp91phox, which is involved in the production of reactive oxygen species to protect against invading pathogens (Robinson et al., 1999; Vignais, 2002). FRO2 was shown to complement the phenotype of an Arabidopsis ferric reductase defective- 1 mutant ( $f r d 1$ ), thus proving that FRO2 encodes the root surface ferric chelate reductase. As expected for an enzyme involved in iron acquisition from the soil, FRO2 is expressed in the root epidermis and is strongly induced by iron limitation (Connolly et al., 2003). Constitutive high-level expression of FRO2 in soybean confers enhanced tolerance to iron deficiency-induced chlorosis (Vasconcelos et al., 2006).

FRO2 belongs to a superfamily of flavocytochromes and is involved in transfer of electrons from the cytosol across the plasma membrane to reduce extracellular ferric iron to ferrous iron. Studies of the topology of FRO2 show that the protein contains eight transmembrane (TM) helices, four of which comprise the highly conserved core of the protein (Schagerlof et al., 2006). This core is conserved throughout the flavocytochrome $b$ family. The large water-soluble domain of FRO2, which contains $\mathrm{NADPH}$, flavin adenine dinucleotide (FAD), and oxidoreductase sequence motifs, is located in the cytosol. FRO2 also contains four highly conserved histidine residues that likely coordinate two intramembranous heme groups that are instrumental in the electron transfer process (Robinson et al., 1999; Schagerlof et al., 2006). Although FRO2 appears to be solely responsible for reduction of ferric iron chelates in the rhizosphere, the Arabidopsis genome encodes a total of eight FRO family members. The seven additional FRO proteins are believed to function as metalloreductases primarily involved in the reduction of iron and possibly copper; here, we highlight new insight into the roles of FRO family members in copper reduction and intracellular metal trafficking.

\section{PLASMA MEMBRANE-LOCALIZED ROOT COPPER REDUCTASES}

Studies of the yeast FRE family have uncovered roles for these proteins in reduction of both iron and copper (Hassett and Kosman, 1995; Georgatsou et al., 1997; Martins et al., 1998). Consistent with their roles in the high-affinity iron and copper uptake systems, their expression is regulated by both iron and copper status. Like their FRE counterparts, Arabidopsis FRO genes are differentially regulated by deficiencies of iron and/or copper (Mukherjee et al., 2006). Studies of FRO2 have suggested that it may have a role in the reduction of $\mathrm{Cu}^{2+}$ to $\mathrm{Cu}^{+}$at the root surface, in addition to its role in iron reduction (Yi and Guerinot, 1996; Robinson et al., 1999). Arabidopsis plants show an increase in root copper reductase activity under iron limitation and $f r d 1$ mutants fail to induce this activity in response to iron limitation (Robinson et al., 1999). However, copper concentrations are not reduced in frd1 mutants, suggesting that reduction of copper by FRO2 is not physiologically relevant; this result opens up the possibility that other FROs function to reduce copper at the root surface. It is possible that copper uptake may proceed without prior reduction of $\mathrm{Cu}^{2+}$ to $\mathrm{Cu}^{+}$, perhaps via a ZRT, IRT-like protein (ZIP)-type transporter. Interestingly, expression of ZIP2 and ZIP4 is upregulated under copper limitation (Wintz et al., 2003). However, stable isotope studies support a reduction-based pathway for copper uptake (Jouvin et al., 2012). Indeed, recent studies have shown that FRO4 and FRO5 act redundantly to reduce copper at the root surface (Bernal et al., 2012)

The SPL7 (SQUAMOSA promoter binding-like7) transcription factor functions as a master regulator of the copper deficiency response in Arabidopsis (Yamasaki et al., 2009). Recently, RNASeq revealed that FRO4 and FRO5 are strongly upregulated in roots under copper limitation. In addition, induction of FRO4 and FRO5 in roots under copper limitation depends on SPL7 (Bernal etal., 2012). FRO4 and FRO5 lie in tandem on chromosome 5 and share high sequence similarity at the amino acid level (Mukherjee et al., 2006). SPL7 has been shown to bind to a CuRE ( $\mathrm{Cu}$ responsive element) in promoters of copper regulated genes (Yamasaki et al., 2004; Yamasaki et al., 2009) similar to its homolog in C. reinhardtii, CCR1 (COPPER RESPONSIVE REGULATOR1; Quinn and Merchant, 1995; Kropat et al., 2005; Sommer et al., 2010). FRO4 and FRO5 each contain GTAC motifs in their upstream promoter regions, suggesting that they may be direct targets of SPL7 (Bernal et al., 2012). fro4, fro5, and fro4fro5 double mutant lines display significant decreases in copper deficiency-inducible copper reductase activity. In addition, use of a fluorescent dye [coppersensor-1 (CS1)] that binds $\mathrm{Cu}^{+}$showed that uptake of $\mathrm{Cu}^{+}$in the fro4 and fro5 single mutants was markedly lower than in wild type plants and fro4fro5 double mutant plants show hardly any detectable $\mathrm{Cu}^{+}$, demonstrating that FRO4 and FRO5 function redundantly as copper reductases in the high affinity copper uptake pathway (Bernal et al., 2012). In addition, although spl7 plants lack expression of FRO4 and FRO5 and corresponding $\mathrm{Cu}$-deficiency inducible root $\mathrm{Cu}$ reductase activity, spl7 does display elevated FRO2 transcript abundance and root ferric chelate reductase activity. These results clearly establish that FRO4 and FRO5 (rather than FRO2) are responsible for reduction of $\mathrm{Cu}$ at the root surface (Bernal et al., 2012). It remains unclear whether FRO4 and FRO5 are involved in Fe homeostasis, however, expression of FRO5 is induced under iron deficiency (Wu et al., 2005; Mukherjee et al., 2006).

\section{PUTATIVE PLASMA MEMBRANE-LOCALIZED LEAF FERRIC REDUCTASE}

Following uptake from the soil, iron must be loaded into the xylem, where it is found as a ferric-citrate complex (RellanAlvarez et al., 2010). How iron is transported into leaf cells remains unknown, but it is thought that Fe(III)-chelates may need to be reduced prior to transport into leaf cells. FRO6 is expressed at high levels in leaves (Mukherjee et al., 2006), and overexpression of FRO6 in tobacco showed that FRO6 can facilitate the reduction of iron in leaves (Li et al., 2011). FRO6 expression is not affected by iron status (Mukherjee et al., 2006). Instead, analysis of FRO6-GUS lines has shown that FRO6 expression is controlled in a light-dependent manner. Indeed, the FRO6 promoter contains several light-responsive elements and etiolated FRO6-GUS seedlings exhibit no FRO6 promoter activity (Feng et al., 2006). Together, these data suggest that FRO6 may function to reduce iron 
in leaves when light is available, perhaps to enable the assembly of new photosynthetic complexes.

\section{INTRACELLULAR METALLOREDUCTASES}

Chloroplasts and mitochondria represent significant sinks for Fe. Indeed, the vast majority of Fe found within leaves is located within chloroplasts. Essential cofactors such as heme and Fe-S clusters are synthesized in chloroplasts and mitochondria. Despite this, the molecular mechanisms by which iron is trafficked to these two organelles are not well understood. Recent studies implicate FRO family members in iron delivery to chloroplasts and mitochondria. Intriguingly, although work in yeast has shown that metalloreductases are important in vacuolar metal homeostasis, to date there is no evidence to support an analogous role in plants.

\section{CHLOROPLASTIC FERRIC REDUCTASE}

Although the precise mechanisms involved in chloroplast iron acquisition are still somewhat murky (Landsberg, 1984; Terry and Abadia, 1986; Bughio et al., 1997; Shikanai et al., 2003), it seems likely that chloroplasts take up both $\mathrm{Fe}(\mathrm{II})$ and $\mathrm{Fe}$ (III) via multiple pathways as observed in modern day cyanobacteria. Free living cyanobacteria have been shown to acquire iron through $\mathrm{Fe}^{2+}$ iron transporters from a pool of $\mathrm{Fe}$ (III)-dicitrate complexes (Katoh etal., 2001) and it is thus clear that some species of cyanobacteria are able to use a reduction-based mechanism for iron uptake (Kranzler et al., 2014). Plant chloroplasts, which are thought to have originated from ancient cyanobacteria, appear to utilize a similar strategy for iron uptake as studies of Arabidopsis FRO7 demonstrate that chloroplasts employ a reduction-based strategy for iron acquisition. FRO7 localizes to chloroplasts and loss of FRO7 function results in a significant reduction in chloroplast surface ferric reductase activity. In addition, fro 7 chloroplasts show a $\sim 30 \%$ reduction in chloroplast $\mathrm{Fe}$ content. fro7 grows poorly on medium lacking sucrose and shows reduced photosynthetic efficiency, consistent with the idea that FRO7 is critical for delivery of Fe for proper assembly of photosynthetic complexes. When sown on alkaline soil, fro7 seeds germinate but the resulting seedlings are severely chlorotic and the plants fail to set seed unless supplemented with excess iron (Jeong et al., 2008). Recent work in sugar beet further supports the existence of a reduction-based mechanism for iron uptake by chloroplasts, as well (Solti et al., 2012).

A presumptive Fe transporter, PIC1, has been identified that localizes to the chloroplast envelope (Duy et al., 2007). Whether FRO7 and PIC1 work together in chloroplast iron uptake currently remains unknown and it is not yet clear whether PIC1 transports ferric or ferrous iron. Other proteins that are presumed to function in chloroplast Fe transport are MAR1 (a homolog of ferroportin 1 and 2), which may transport an iron chelator (Conte etal., 2009), MFL1/2 [which resemble mitoferrins but function in chloroplasts; (Tarantino et al., 2011) and NAP14 (Shimoni-Shor etal., 2010)]. In addition, a chloroplast-and mitochondria-localized NEET-type protein was recently identified which may be involved in Fe-S cluster transfer to apoproteins (Nechushtai et al., 2012).

\section{PUTATIVE MITOCHONDRIAL FERRIC REDUCTASES}

Studies in Arabidopsis have identified a putative iron-chaperone (Busi etal., 2006; Vazzola etal., 2007) and putative mitochondrial effluxer proteins involved in iron metabolism (Kushnir et al., 2001; Chen et al., 2007). In addition, a recent report described the identification of a mitochondrial Fe transporter in rice (MIT1) which is essential for plant growth (Bashir et al., 2011). Despite this, we are far from a comprehensive understanding of mitochondrial Fe homeostasis (Nouet et al., 2011; Vigani et al., 2013). Although two Arabidopsis metalloreductases (FRO3 and FRO8) have been predicted to localize to mitochondrial membranes, neither one has been functionally characterized. A mitochondrial proteomics study has placed FRO8 at the mitochondrial membrane (Heazlewood et al., 2004). The expression patterns of FRO3 and FRO8 are largely non-overlapping, suggesting that they do not function redundantly (Jain and Connolly, 2013). Little information is available for FRO8 but its expression is concentrated in the vasculature of senescing leaves (Wu et al., 2005). FRO3 is expressed most highly in the vasculature of young seedlings and its expression is strongly induced under iron deficiency; for this reason, FRO3 has been widely used as an iron deficiency marker (Mukherjee et al., 2006; Tarantino et al., 2010). Interestingly, FRO3 expression is negatively regulated by the basic helix loop helix (bHLH) transcription factor PYE (POPEYE); PYE appears to control a pericycle-specific Fe deficiency response in roots (Dinneny et al., 2008; Long et al., 2010). FRO3 expression also is responsive to copper status (Mukherjee et al., 2006; Yamasaki et al., 2009). Despite this, the roles of FRO3 and FRO8 remain unclear. It is interesting to note that although the yeast metalloreductase FRE5 localizes to mitochondria (Sickmann et al., 2003), there are no reports to date that demonstrate a role for a metalloreductase in mitochondria in any organism.

\section{VACUOLAR IRON TRAFFICKING}

Acidic compartments like vacuoles have a relatively oxidizing atmosphere as compared to the cytosol. In yeast, iron in vacuoles is largely present as ferric polyphosphate complexes (Raguzzi et al., 1988). The remobilization of iron from the yeast vacuolar compartment is mediated by the FRE6 ferric chelate reductase (Singh et al., 2007). FRE6 also plays a role in copper remobilization from vacuoles; reduced copper is subsequently exported to the cytosol via CTR2 (copper transporter 2; Rees and Thiele, 2007). Vacuolar iron transporters have been reported in plants; Arabidopsis vacuolar iron transporter (VIT1), transports iron into the organelle while NRAMP3 (natural resistance against microbial pathogens3) and NRAMP4 mediate the export of iron (Lanquar et al., 2005; Kim et al., 2006). However, no vacuolar metalloreductases have been reported in plants, to date.

\section{CONCLUSION}

Plants require iron and copper for vital processes such as photosynthesis, respiration, and nitrogen fixation. While it has been known for some time that ferric chelate reductases play a vital role in iron uptake from the soil by all plant species except for grasses, other roles for FROs in metal homeostasis have only recently emerged. Indeed, new studies have shown that FROs are important for copper acquisition from the soil (Figure 1) and for intracellular 


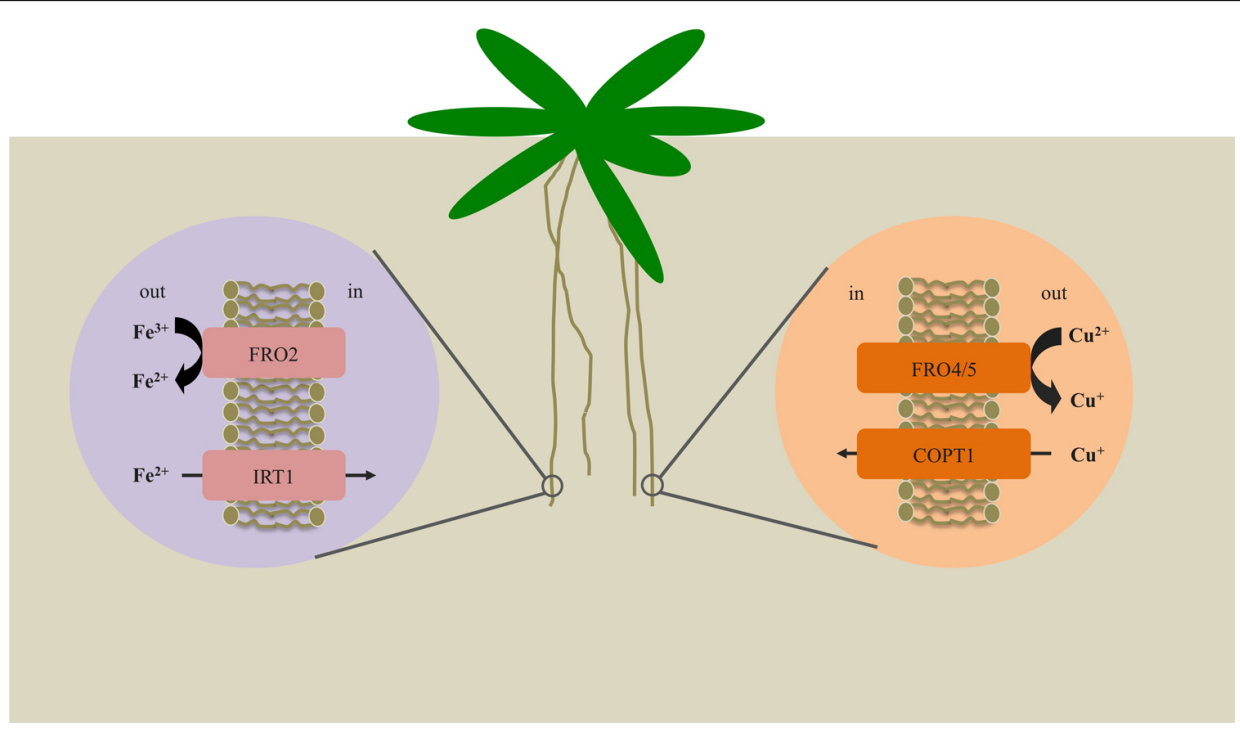

FIGURE 1 | Mechanisms for iron and copper uptake by Arabidopsis roots. Under iron-deficient conditions, expression of FRO2 and IRT1 is enhanced. $\mathrm{FRO} 2$ serves to reduce solubilized $\mathrm{Fe}^{3+}$ to $\mathrm{Fe}^{2+}$, which is then transported across the root plasma membrane via IRT1. Under copper-deficient conditions, FRO4 and FRO5 are highly expressed in the roots and function to reduce $\mathrm{Cu}^{2+}$ to $\mathrm{Cu}^{+}$prior to uptake by COPT1.

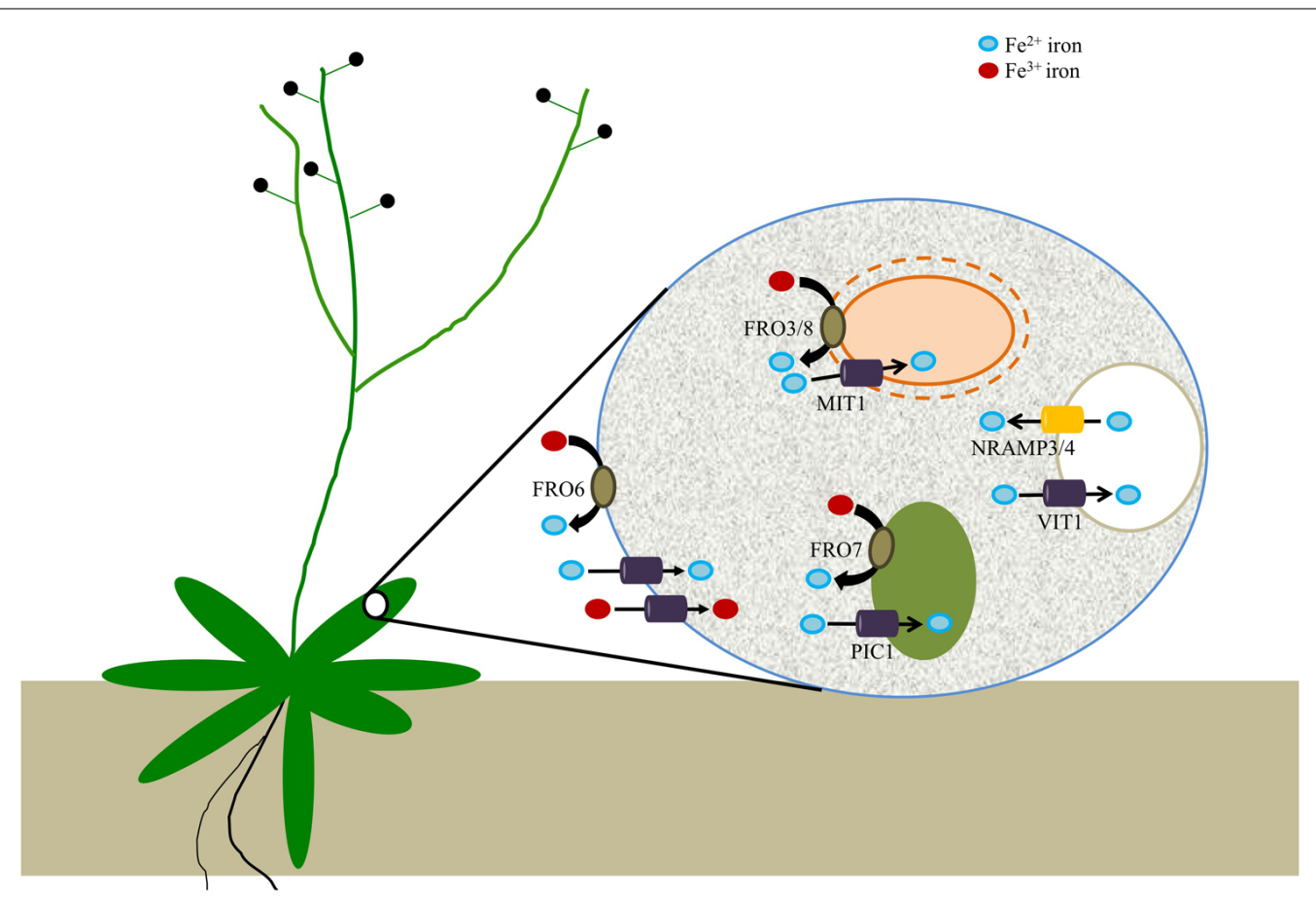

FIGURE 2 | Arabidopsis ferric reductases and transporters that contribute to cellular iron homeostasis. Evidence suggests that FRO6 functions to reduce $\mathrm{Fe}^{3+}$ to $\mathrm{Fe}^{2+}$ at the cell surface of leaf cells; $\mathrm{Fe}^{2+}$ is subsequently transported across the membrane via an unknown transporter(s), while other unknown transporters may be involved in the uptake of $\mathrm{Fe}^{3+}$. Iron is then trafficked to a set of intracellular organelles.
Chloroplasts utilize a reduction-based mechanism for iron acquisition via $\mathrm{FRO7}$, whereas FRO3 and FRO8 may serve an analogous function in mitochondria. PIC1 serves as a chloroplast iron transporter while rice MIT mediates iron uptake by mitochondria. Although there is not yet any evidence for vacuolar metalloreductases in plants, it is known that VIT1 is important for iron uptake by vacuoles while NRAMP3/4 function in vacuolar Fe efflux. 
distribution of $\mathrm{Fe}$ (Figure 2). Together, these studies have shed considerable light on the molecular mechanisms employed by plants to maintain $\mathrm{Fe}$ and $\mathrm{Cu}$ homeostasis. In addition, this new knowledge should facilitate novel strategies aimed at improving crop yields on nutrient-poor soils and biofortification of plant foods to help ameliorate nutrient deficiencies in humans. Future studies will likely focus on the precise roles of mitochondrial FROs in mitochondrial metal metabolism. Furthermore, our understanding of iron trafficking within cells is severely hampered by our limited understanding of the various subcellular iron pools. New tools that provide insight into the redox status and types of iron species found in each of the various cellular compartments will go a long way toward the development of a comprehensive understanding of iron metabolism in plants.

\section{ACKNOWLEDGMENT}

The authors gratefully acknowledge support from the US NSF (IOS 0919739).

\section{REFERENCES}

Bashir, K., Ishimaru, Y., Shimo, H., Nagasaka, S., Fujimoto, M., Takanashi, H., et al. (2011). The rice mitochondrial iron transporter is essential for plant growth. Nat. Commun. 2, 1-7. doi: 10.1038/ncomms1326

Bernal, M., Casero, D., Singh, V., Wilson, G. T., Grande, A., Yang, H., et al. (2012). Transcriptome sequencing identifies SPL7-regulated Cu acquisition genes FRO4/FRO5 and the $\mathrm{Cu}$ dependence of Fe homeostasis in Arabidopsis. Plant Cell 24, 738-761. doi: 10.1105/tpc.111.090431

Bughio, N., Takahashi, M., Yoshimura, E., Nishizawa, N. K., and Mori, S. (1997). Light-dependent iron transport into isolated barley chloroplasts. Plant Cell Physiol. 38, 101-105. doi: 10.1093/oxfordjournals.pcp.a 029079

Busi, M. V., Maliandi, M. V., Valdez, H., Clemente, M., Zabaleta, E. J., Araya, A., et al. (2006). Deficiency of Arabidopsis thaliana frataxin alters activity of mitochondrial Fe-S proteins and induces oxidative stress. Plant J. 48, 873-882. doi: 10.1111/j.1365-313X.2006.02923.x

Chen, S., Sanchez-Fernandez, R., Lyver, E. R., Dancis, A., and Rea, P. A. (2007). Functional characterization of AtATM1, AtATM2 and AtATM3, a subfamily of Arabidopsis half-molecule ABC transporters implicated in iron homeostasis. J. Biol. Chem. 282, 21561-21571. doi: 10.1074/jbc.M702383200

Connolly, E. L., Campbell, N., Grotz, N., Prichard, C. L., and Guerinot, M. L. (2003). Overexpression of the FRO2 iron reductase confers tolerance to growth on low iron and uncovers post-transcriptional control. Plant Physiol. 133, 1102-1110. doi: 10.1104/pp.103.025122

Conte, S., Stevenson, D., Furner, I., and Lloyd, A. (2009). Multiple antibiotic resistance in Arabidopsis is conferred by mutations in a chloroplast-localized transport protein. Plant Physiol. 151, 559-573. doi: 10.1104/pp.109.143487

Curie, C., Panaviene, Z., Loulergue, C., Dellaporta, S. L., Briat, J. F., and Walker, E. L. (2001). Maize yellow stripel encodes a membrane protein directly involved in Fe(III) uptake. Nature 409, 346-349. doi: 10.1038/35053080

Dinneny, J. R., Long, T. A., Wang, J. Y., Jung, J. W., Mace, D., Pointer, S., et al. (2008). Cell identity mediates the response of Arabidopsis roots to abiotic stress. Science 320, 942-945. doi: 10.1126/science.1153795

Duy, D., Wanner, G., Meda, A. R., Von Wiren, N., Soll, J., and Philippar, K. (2007). PIC1, an ancient permease in Arabidopsis chloroplasts, mediates iron transport. Plant Cell 19, 986-1006. doi: 10.1105/tpc.106.047407

Eckhardt, U., and Buckhout, T. J. (1998). Iron assimilation in Chlamydomonas reinhardtii involves ferric reduction and is similar to strategy I higher plants. J. Exp. Bot. 9, 1219-1226.

Eide, D., Broderius, M., Fett, J., and Guerinot, M. L. (1996). A novel iron-regulated metal transporter from plants identified by functional expression in yeast. Proc. Natl. Acad. Sci. U.S.A. 93, 5624-5628. doi: 10.1073/pnas.93.11.5624

Feng, H., An, F., Zhang, S., Ji, Z., Ling, H. Q., and Zuo, J. (2006). Light-regulated, tissue-specific, and cell differentiation-specific expression of the Arabidopsis Fe(III)-chelate reductase gene AtFRO6. Plant Physiol. 140, 1345-1354. doi: 10.1104/pp.105.074138I
Georgatsou, E., Mavrogiannis, L. A., Fragiadakis, G. S., and Alexandraki, D. (1997). The yeast Fre1p/Fre2p cupric reductases facilitate copper uptake and are regulated by the copper-modulated Maclp activator. J. Biol. Chem. 272, 13786-13792. doi: 10.1074/jbc.272.21.13786

Guerinot, M. L., and Yi, Y. (1994). Iron: nutritious, noxious, and not readily available. Plant Physiol. 104, 815-820. doi:10.1104/pp.104.3.815

Halliwell, B., and Gutteridge, J. M. C. (1992). Biologically relevant metal ion-dependent hydroxyl radical generation. FEBS Lett. 307, 108-112. doi: 10.1016/0014-5793(92)80911-Y

Hassett, R., and Kosman, D. J. (1995). Evidence for Cu(II) reduction as a component of copper uptake by Saccharomyces cerevisiae. J. Biol. Chem. 270, 128-134. doi: $10.1074 /$ jbc. 270.1 .128

Heazlewood, J. L., Tonti-Filippini, J. S., Gout, A. M., Day, D. A., Whelan, J., and Millar, A. H. (2004). Experimental analysis of the Arabidopsis mitochondrial proteome highlights signaling and regulatory components, provides assessment of targeting prediction programs, and indicates plant-specific mitochondrial proteins. Plant Cell 16, 241-256. doi: 10.1105/tpc.016055

Jain, A., and Connolly, E. L. (2013). Mitochondrial iron transport and homeostasis in plants. Front. Plant Sci. 4:348. doi: 10.3389/fpls.2013.00348

Jeong, J., Cohu, C., Kerkeb, L., Pilon, M., Connolly, E. L., and Guerinot, M. L. (2008). Chloroplast $\mathrm{Fe}$ (III) chelate reductase activity is essential for seedling viability under iron limiting conditions. Proc. Natl. Acad. Sci. U.S.A. 105, 10619-10624. doi: 10.1073/pnas.0708367105

Jouvin, D., Weiss, D. J., Mason, T. F., Bravin, M. N., Louvat, P., Zhao, F., et al. (2012). Stable isotopes of $\mathrm{Cu}$ and $\mathrm{Zn}$ in higher plants: evidence for $\mathrm{Cu}$ reduction at the root surface and two conceptual models for isotopic fractionation processes. Environ. Sci. Technol. 46, 2652-2660. doi: 10.1021/es202587m

Katoh, H., Hagino, N., Grossman, A. R., and Ogawa, T. (2001). Genes essential to iron transport in the cyanobacterium Synechocystis sp. strain PCC 6803. J. Bacteriol. 183, 2779-2784. doi: 10.1128/JB.183.9.2779-2784.2001

Kim, S. A., Punshon, T., Lanzirotti, A., Li, L., Alonso, J. M., Ecker, J. R., et al. (2006). Localization of iron in Arabidopsis seed requires the vacuolar membrane transporter VIT1. Science 314, 1295-1298. doi: 10.1126/science.1132563

Kranzler, C., Lis, H., Finkel, O. M., Schmetterer, G., Shaked, Y., and Keren, N. (2014). Coordinated transporter activity shapes high-affinity iron acquisition in cyanobacteria. ISME J. 8, 409-417. doi: 10.1038/ismej.2013.161

Kropat, J., Tottey, S., Birkenbihl, R. P., Depege, N., Huijser, P., and Merchant, S. (2005). A regulator of nutritional copper signaling in Chlamydomonas is an SBP domain protein that recognizes the GTAC core of copper response element. Proc. Natl. Acad. Sci. U.S.A. 102, 18730-18735. doi: 10.1073/pnas. 0507693102

Kushnir, S., Babiychuk, E., Storozhenko, S., Davey, M. W., Papenbrock, J., De Rucke, R., et al. (2001). A mutation of the mitochondrial ABC transporter Stal leads to dwarfism and chlorosis in the Arabidopsis mutant starik. Plant Cell 13, 89-100.

Landsberg, E. (1984). Regulation of iron-stress-response by whole-plant activity. J. Plant Nutr. 7, 609-621. doi: 10.1080/01904168409363226

Lanquar, V., Lelievre, F., Bolte, S., Hames, C., Alcon, C., Neumann, D., et al. (2005). Mobilization of vacuolar iron by AtNRAMP3 and AtNRAMP4 is essential for seed germination on low iron. EMBO J. 24, 4041-4051. doi: 10.1038/sj.emboj. 7600864

Li, L., Cheng, X., and Ling, H. Q. (2004). Isolation and characterization of Fe(III)chelate reductase gene LeFRO1 in tomato. Plant Mol. Biol. 54, 125-136. doi: 10.1023/B:PLAN.0000028774.82782.16

Li, L. Y., Cai, Q. Y., Yu, D. S., and Guo, C. H. (2011). Overexpression of AtFRO6 in transgenic tobacco enhances ferric chelate reductase activity in leaves and increases tolerance to iron-deficiency chlorosis. Mol. Biol. Rep. 38, 3605-3613. doi: 10.1007/s11033-010-0472-9I

Long, T. A., Tsukagoshi, H., Busch, W., Lahner, B., Salt, D. E., and Benfey, P. N. (2010). The bHLH transcription factor POPEYE regulates response to iron deficiency in Arabidopsis roots. Plant Cell 22, 2219-2236. doi: 10.1105/tpc.110.074096

Martins, L. J., Jensen, L. T., Simon, J.R., Keller, G. L., and Winge, D. R. (1998). Metalloregulation of FRE1 and FRE2 homologs in Saccharomyces cerevisiae. J. Biol. Chem. 273, 23716-23721. doi: 10.1074/jbc.273.37.23716

Mukherjee, I., Campbell, N. H., Ash, J. S., and Connolly, E. L. (2006). Expression profiling of the Arabidopsis ferric chelate reductase (FRO) gene family reveals differential regulation by iron and copper. Planta 223, 1178-1190. doi: 10.1007/s00425-005-0165-0 
Nechushtai, R., Conlan, A. R., Harir, Y., Song, L., Yogev, O., Eisenberg-Domovich, Y., etal. (2012). Characterization of Arabidopsis NEET reveals an ancient role for NEET proteins in iron metabolism. Plant Cell 24, 2139-2154. doi: 10.1105/tpc.112.097634

Nouet, C., Motte, P., and Hanikenne, M. (2011). Chloroplastic and mitochondrial metal homeostasis. Trends Plant Sci. 16, 395-404. doi 10.1016/j.tplants.2011.03.005

Quinn, J. M., and Merchant, S. (1995). Two copper-responsive elements associated with the Chlamydomonas Cyc6 gene function as targets for transcriptional activators. Plant Cell 7, 623-628. doi: 10.1105/tpc.7.5.623

Raguzzi, F., Lesuisse, E., and Crichton, R. R. (1988). Iron storage in Saccharomyces cerevisiae. FEBS Lett. 231, 253-258. doi: 10.1016/0014-5793(88)80742-7

Rees, E. M., and Thiele, D. J. (2007). Identification of a vacuole-associated metalloreductase and its role in Ctr2-mediated intracellular copper mobilization. J. Biol. Chem. 282, 21629-21638. doi: 10.1074/jbc.M703397200

Rellan-Alvarez, R., Giner-Martinez-Sierra, J., Orduna, J., Orera, I., RodriguezCastrillon, J. A., Garcia-Alonso, J. I., et al. (2010). Identification of a tri-iron(III), tri-citrate complex in the xylem sap of iron-deficient tomato resupplied with iron: new insights into plant iron long-distance transport. Plant Cell Physiol. 51, 91-102. doi: 10.1093/pcp/pcp170

Robinson, N. J., Procter, C. M., Connolly, E. L., and Guerinot, M. L. (1999). A ferric-chelate reductase for iron uptake from soils. Nature 397, 694-697. doi: $10.1038 / 17800$

Santi, S., and Schmidt, W. (2009). Dissecting iron deficiency-induced proton extrusion in Arabidopsis roots. New Phytol. 183, 1072-1084. doi: 10.1111/j.14698137.2009.02908.x

Schagerlof, U., Wilson, G., Hebert, H., Al-Karadaghi, S., and Hagerhall, C. (2006). Transmembrane topology of FRO2, a ferric chelate reductase from Arabidopsis thaliana. Plant Mol. Biol. 62, 215-221. doi: 10.1007/s11103-006-9015-0

Shikanai, T., Muller-Moule, P., Munekage, Y., Niyogi, K. K., and Pilon, M. (2003). PAA1, a P-type ATPase of Arabidopsis, functions in copper transport in chloroplasts. Plant Cell 15, 1333-1346. doi: 10.1105/tpc.011817

Shimoni-Shor, E., Hassidim, M., Yuval-Naeh, N., and Keren, N. (2010). Disruption of Nap14, a plastid-localized non-intrinsic ABC protein in Arabidopsis thaliana results in the over-accumulation of transition metals and in aberrant chloroplast structures. Plant Cell Environ. 33, 1029-1038. doi: 10.1111/j.13653040.2010.02124.x

Sickmann, A., Reinders, J., Wagner, Y., Joppich, C., Zahedi, R., Meyer, H. E., et al. (2003). The proteome of Saccharomyces cerevisiae mitochondria. Proc. Natl. Acad. Sci. U.S.A. 100, 13207-13212. doi: 10.1073/pnas.2135385100

Singh, A., Kaur, N., and Kosman, D. J. (2007). The metalloreductase Fre6p in Fe-efflux from the yeast vacuole. J. Biol. Chem. 282, 28619-28626. doi: 10.1074/jbc.M703398200

Solti, Á., Kovács, K., Basa, B., Vértes, A., Sárvári, É., and Fodor, F. (2012). Uptake and incorporation of iron in sugar beet chloroplasts. Plant Physiol. Biochem. 52, 91-97. doi: 10.1016/j.plaphy.2011.11.010

Sommer, F., Kropat, J., Malasarn, D., Grossoehme, N. E., Chen, X., Giedroc, D. P., et al. (2010). The CRR1 nutritional copper sensor in Chlamydomonas contains two distinct metal-responsive domains. Plant Cell 22, 4098-4113. doi: 10.1105/tpc.110.080069

Tarantino, D., Morandini, P., Ramirez, L., Soave, C., and Murgia, I. (2011). Identification of an Arabidopsis mitoferrinlike carrier protein involved in Fe metabolism. Plant Physiol. Biochem. 49, 520-529. doi: 10.1016/j.plaphy.2011.02.003

Tarantino, D., Santo, N., Morandini, P., Casagrande, F., Braun, H. P., Heinemeyer, J., et al. (2010). AtFer4 ferritin is a determinant of iron homeostasis in Arabidopsis thaliana heterotrophic cells. J. Plant Physiol. 167, 1598-1605. doi: 10.1016/j.jplph.2010.06.020

Terry, N., and Abadia, J. (1986). Function of iron in chloroplasts. J. Plant Nutr. 9, 609-646. doi: 10.1080/01904168609363470
Vasconcelos, M., Eckert, H., Arahana, V., Graef, G., Grusak, M. A., and Clemente, T. (2006). Molecular and phenotypic characterization of transgenic soybean expressing the Arabidopsis ferric chelate reductase gene, FRO2. Planta 224, 1116-1128. doi: 10.1007/s00425-006-0293-1

Vazzola, V., Losa, A., Soave, C., and Murgia, I. (2007). Knockout of frataxin gene causes embryo lethality in Arabidopsis. FEBS Lett. 581, 667-672. doi: 10.1016/j.febslet.2007.01.030

Vert, G., Grotz, N., Dedaldechamp, F., Gaymard, F., Guerinot, M. L., Briat, J. F., et al. (2002). IRT1, an Arabidopsis transporter essential for iron uptake from the soil and for plant growth. Plant Cell 14, 1223-1233. doi: 10.1105/tpc. 001388

Vigani, G., Zocchi, G., Bashir, K., Philippar, K., and Briat, J. F. (2013). Signals from chloroplasts and mitochondria for iron homeostasis regulation. Trends Plant Sci. 18, 305-311. doi: 10.1016/j.tplants.2013.01.006

Vignais, P. V. (2002). The superoxide-generating NADPH oxidase: structural aspects and activation mechanism. Cell Mol. Life Sci. 59, 1428-1459. doi: 10.1007/s00018002-8520-9

Walker, E. L., and Connolly, E. L. (2008). Time to pump iron: iron-deficiencysignaling mechanisms of higher plants. Curr. Opin. Plant Biol. 11, 530-535.

Waters, B. M., Blevins, D. G., and Eide, D. J. (2002). Characterization of FRO1, a pea ferric-chelate reductase involved in root iron acquisition. Plant Physiol. 129, 85-94. doi: 10.1104/pp.010829

Wintz, H., Fox, T., Wu, Y. Y., Feng, V., Chen, W., Chang, H. S., et al. (2003). Expression profiles of Arabidopsis thaliana in mineral deficiencies reveal novel transporters involved in metal homeostasis. J. Biol. Chem. 278, 47644-47653. doi: 10.1074/jbc.M309338200

Wu, H., Li, L., Du, J., Yuan, Y., Cheng, X., and Ling, H. Q. (2005). Molecular and biochemical characterization of the $\mathrm{Fe}$ (III) chelate reductase gene family in Arabidopsis thaliana. Plant Cell Physiol. 46, 1505-1514. doi: 10.1093/pcp/ pcil63

Yamasaki, H., Hayashi, M., Fukazawa, M., Kobayashi, Y., and Shikanai, T. (2009). SQUAMOSA promoter binding protein-like7 is a central regulator for copper homeostasis in Arabidopsis. Plant Cell 21, 347-361. doi: 10.1105/tpc.108. 060137

Yamasaki, K., Kigawa, T., Inoue, M., Tateno, M., Yamasaki, T., Yabuki, T., et al. (2004). A novel zinc-binding motif revealed by solution structures of DNAbinding domains of Arabidopsis SBP-family transcription factors. J. Mol. Biol. 337, 49-63. doi: 10.1016/j.jmb.2004.01.015

Yi, Y., and Guerinot, M. L. (1996). Genetic evidence that induction of root Fe(III) chelate reductase activity is necessary for iron uptake under iron deficiency. Plant J. 10, 835-844. doi: 10.1046/j.1365-313X.1996.10050835.x

Conflict of Interest Statement: The authors declare that the research was conducted in the absence of any commercial or financial relationships that could be construed as a potential conflict of interest.

Received: 28 January 2014; paper pending published: 14 February 2014; accepted: 02 March 2014; published online: 21 March 2014.

Citation: Jain A, Wilson GT and Connolly EL (2014) The diverse roles of FRO family metalloreductases in iron and copper homeostasis. Front. Plant Sci. 5:100. doi: 10.3389/fpls.2014.00100

This article was submitted to Plant Nutrition, a section of the journal Frontiers in Plant Science.

Copyright (c) 2014 Jain, Wilson, and Connolly. This is an open-access article distributed under the terms of the Creative Commons Attribution License (CC BY). The use, distribution or reproduction in other forums is permitted, provided the original author(s) or licensor are credited and that the original publication in this journal is cited, in accordance with accepted academic practice. No use, distribution or reproduction is permitted which does not comply with these terms. 